ABC method 13

Biomarker 32

Cancer screening 13

Colitis-associated cancer 32, 40

Conventional white light endoscopic features 47

Crohn's disease 66

Dysphagia 7

Early gastric cancer 53

Endoscopic submucosal dissection 53 Eosinophile 7

Eotaxin 7

Eradication of Helicobacter pylori 19

Firmicutes 59

Food allergy 7

Gastric cancer 13, 19

Gastric mucosal atrophy 24

Gastroesophageal reflux disease 24

Helicobacter pylori 7, 13, 19, 24

Hiatus hernia 24

Indication criteria 53

Inflammatory bowel diseases 40
Long-term survival 53

Microbiota 59

Mucosal healing 32

Myosin light chain kinase 40

Non-curative resection 53

Non-erosive reflux disease 24

Pepsinogen 13

Peptic ulcer 19

Prostaglandin E-major urinary metabolite 32

Reflux esophagitis 19, 24

Rutgeerts' score 66

\section{SESCD 66}

Short-chain fatty acids 59

Short-segment Barrett's esophagus 47 Superficial Barrett's esophageal adenocarcinoma 47

Tight junction 40

Tissue remodeling 32

Tumor necrosis factor 40

Ulcerative colitis 32 